\title{
ON ABSOLUTELY CONVERGENT DIRICHLET SERIES
}

\author{
D. A. EDWARDS ${ }^{1}$
}

1. Suppose that $f(s) \equiv f(\sigma+i t)$ is defined for all $\sigma \geqq 0$ as the sum of an ordinary Dirichlet series $\sum_{n=1}^{\infty} a_{n} n^{-8}$ such that $\sum_{1}^{\infty}\left|a_{n}\right|<\infty$. Then, by characterizing the regular elements of a certain Banach algebra associated with the set of all such functions, Hewitt and Williamson [3] have shown that $[f(s)]^{-1}$ can be expressed as the sum of an ordinary Dirichlet series

$$
[f(s)]^{-1}=\sum_{n=1}^{\infty} b_{n} n^{-s} \quad(\sigma \geqq 0),
$$

satisfying $\sum_{1}^{\infty}\left|b_{n}\right|<\infty$, if and only if $f(s)$ is bounded away from zero in the half-plane $\sigma \geqq 0$. This discovery amounts to a determination of the spectrum of the Banach-algebra element associated with $f(s)$, and it thus makes available for the theory of ordinary Dirichlet series the well-known theorem of Gelfand on analytic functions of Banach-algebra elements (see $\$ 24 \mathrm{D}$ of [7]) and its generalization, the Silov-Arens-Calderón theorem (Theorem 3.3 of $[1]$ ).

This paper is intended to show that the theorem of Hewitt and Williamson, together with a similar theorem for general Dirichlet series $\sum_{n=0}^{\infty} a_{n} e^{-\lambda_{n} s}$, can be deduced by an elementary argument from a theorem due to Phillips (stated below, in a weakened form, as Theorem 1). The possibility of such an argument arises from the circumstance that the Banach algebra considered by Hewitt and Williamson (and likewise each of the algebras $\mathfrak{A}_{\Gamma}$ introduced below) can be enlarged in a certain way without decreasing the spectra of the original elements. No attempt however will be made to examine the maximal ideal spaces of the algebras which occur (the reader who wishes to pursue the subject is referred to the writings $[4 ; 5]$ of Hewitt and Zuckerman): the object here is to show what can be done without introducing new machinery.

2. Let $\beta$ be the $\sigma$-algebra of Borel subsets of the closed half-line $[0, \infty)$ and let $\mathfrak{P}$ be the set of all complex-valued functions on $B$ of the form

$$
\alpha(E)=\sum_{r=0}^{3} i^{r} \alpha^{(r)}(E)
$$

Received by the editors December 10, 1956 and, in revised form, April 22, 1957.

1 The author is a Research Associate of the National Science Foundation. 
where the $\alpha^{(r)}$ are totally finite countably additive non-negative measures on $B$. For each $\alpha \in \mathfrak{A}$ let the norm $\|\alpha\|$ be defined by

$$
\|\alpha\|=\sup \sum_{n=1}^{N}\left|\alpha\left(E_{n}\right)\right|,
$$

where the supremum is taken over all representations of $[0, \infty)$ as a finite disjoint union $\bigcup_{n=1}^{N} E_{n}$ of sets $E_{n} \in \mathbb{B}$. Each $\alpha \in \mathfrak{A}$ has a unique decomposition into a sum of three components in $\mathfrak{A}$ :

$$
\alpha=\alpha_{1}+\alpha_{2}+\alpha_{3},
$$

where $\alpha_{1}$ is absolutely continuous with respect to Lebesgue measure, $\alpha_{2}$ is atomic and $\alpha_{3}$ is singular but nonatomic. $\mathfrak{P}$ is a Banach space when the elementary operations are defined in the natural way, and if the product of $\alpha, \beta \in \mathfrak{A}$ is defined to be their convolution $\alpha * \beta \in \mathfrak{A}$, namely the element of $\mathfrak{A}$ given by

$$
(\alpha * \beta)(E)=\int_{0}^{\infty} \alpha(E-\xi) \beta(d \xi)
$$

where

$$
\alpha(E-\xi) \equiv \alpha(\{\eta: \eta \geqq 0, \xi+\eta \in E\}),
$$

then $\mathfrak{A}$ is actually a commutative Banach algebra with a unit element $e$. For a fuller discussion of this algebra the paper of Phillips [8] and book of Hille and Phillips [6] may be consulted. A proof of the following theorem has been sketched in [8] (a full account appears in $[6])$.

Theorem 1 (Phillips). ${ }^{2}$ Suppose that the singular nonatomic component of $\alpha \in \mathfrak{A}$ is the zero element $\theta$ of $\mathfrak{A}$. Then $\alpha$ has an inverse in $\mathfrak{A}$ if and only if

$$
\inf _{\sigma \geqq 0}\left|\int_{0}^{\infty} e^{-s \lambda} \alpha(d \lambda)\right|>0
$$

Now suppose that $\alpha$ is purely atomic $\left(\alpha=\alpha_{2}\right)$ and that $\alpha * \beta=e$, where $\beta$ has the decomposition $\beta=\beta_{1}+\beta_{2}+\beta_{3}$ corresponding to (2). Then the decomposition of $\alpha * \beta$ is just the sum

$$
\alpha_{2} * \beta_{1}+\alpha_{2} * \beta_{2}+\alpha_{2} * \beta_{3}=\alpha * \beta=e .
$$

Clearly $\alpha_{2} * \beta_{1}=\alpha_{2} * \beta_{3}=\theta$ and $\alpha_{2} \neq \theta$. But $\mathfrak{A}$ has no divisors of zero.

2 I am indebted to Professor E. Hille for drawing my attention to this theorem and for subsequently encouraging me to write this paper. I also thank the referee and Professor S. Kakutani for their criticisms and suggestions. 
(This assertion is a consequence of the uniqueness theorem for Laplace-Stieltjes transforms and the fact that $\int_{0}^{\infty} e^{-s \lambda} \gamma(d \lambda)(\gamma \in \mathfrak{A})$ is analytic for $\sigma>0$.) Consequently $\beta_{1}=\beta_{3}=\theta$, and so $\beta=\beta_{2}$; that is, $\beta$ is also purely atomic. The Laplace-Stieltjes transforms corresponding to $\alpha$ and $\beta$ may therefore be expressed as sums

$$
\int_{0}^{\infty} e^{-s \lambda} \alpha(d \lambda)=\sum_{n=0}^{\infty} a_{n} e^{-\lambda_{n} \varepsilon}, \quad \int_{0}^{\infty} e^{-s \mu} \beta(d \mu)=\sum_{n=0}^{\infty} b_{n} e^{-\mu_{n} \varepsilon},
$$

for $\sigma \geqq 0$, where $\sum_{0}^{\infty}\left|a_{n}\right|<\infty, \sum_{0}^{\infty}\left|b_{n}\right|<\infty$ and where $\left\{\lambda_{n}\right\},\left\{\mu_{n}\right\}$ are both sequences of distinct points in $[0, \infty)$. One can evidently suppose that $\lambda_{0}=\mu_{0}=0$ and that $a_{n} \neq 0, b_{n} \neq 0(n=1,2, \cdots)$. Suppose also that $J$ is the set of non-negative integers.

Theorem 2. A purely atomic measure $\alpha \in \mathfrak{A}$ has an inverse in $\mathfrak{A}$ if and only if its Laplace-Stieltjes transform $\sum_{n=0}^{\infty} a_{n} e^{-\lambda_{n} s}$ satisfies the condition

$$
\inf _{\sigma \geqq 0}\left|\sum_{n=0}^{\infty} a_{n} e^{-\lambda_{n} s}\right|>0 .
$$

In this case its inverse $\beta$ is also purely atomic and the Laplace-Stieltjes transform $\sum_{n=0}^{\infty} b_{n} e^{-\mu_{n 8}}$ of $\beta$ has the property that its exponents $\mu_{n}$ all belong to the J-module generated by the set $\left\{\lambda_{n}: n \in J\right\}$.

If $f(s)=\sum_{n=0}^{\infty} a_{n} e^{-\lambda_{n s}}(\sigma \geqq 0)$ then for each $\delta>0$

$$
\begin{aligned}
\left|f(s)-a_{0}\right| & \leqq \sum_{n=1}^{\infty}\left|a_{n}\right| e^{-\lambda_{n} \sigma} \\
& \leqq \sum_{0<\lambda_{n}<\delta}\left|a_{n}\right|+e^{-\delta \sigma} \sum_{\lambda_{n} \geqq \delta}\left|a_{n}\right| .
\end{aligned}
$$

The first term on the right is $o(1)$ as $\delta \downarrow 0$. For fixed $\delta>0$ the final term is $o(1)$ as $\sigma \rightarrow \infty$. It follows that $f(s) \rightarrow a_{0}$ uniformly in $t$ as $\sigma \rightarrow \infty$, and hence that condition (3) cannot be satisfied unless $a_{0} \neq 0$. If $a_{0} \neq 0$ then the argument just given shows that there is a $\sigma_{0}>0$ for which

$$
\left|\sum_{n=1}^{\infty} a_{n} e^{-\lambda_{n} s}\right| \leqq \sum_{n=1}^{\infty}\left|a_{n}\right| e^{-\lambda_{n} \sigma}<\frac{1}{2}\left|a_{0}\right| \text { when } \sigma \geqq \sigma_{0} .
$$

Then in the half-plane $\sigma \geqq \sigma_{0}$ we have

(6) $\frac{1}{f(s)}=\frac{1}{a_{0}+\sum_{n=1}^{\infty} a_{n} e^{-\lambda_{n} \varepsilon}}=a_{0}^{-1}\left[1+\sum_{m=1}^{\infty}(-1)^{m}\left(a_{0}^{-1} \sum_{n=1}^{\infty} a_{n} e^{-\lambda_{n} s}\right)^{m}\right]$, 
the geometric series being convergent on account of the inequality (5). A straightforward absolute-convergence argument now allows us to rearrange the terms in (6) to obtain

$$
\frac{1}{f(s)}=\sum_{n=0}^{\infty} c_{n} e^{-\nu_{n} s} \quad\left(\sigma \geqq \sigma_{0}\right),
$$

where $\left\{\nu_{n}: n=0,1,2, \cdots\right\}$ is a sequence of distinct points in the $J$-module generated by $\left\{\lambda_{n}: n=0,1,2, \cdots\right\}$. Since $[f(s)]^{-1}$ is also equal to $\sum_{n=0}^{\infty} b_{n} e^{-\mu_{n} s}$ for $\sigma \geqq \sigma_{0}$, the final assertion of Theorem 2 now follows from the uniqueness theorem for Laplace-Stieltjes transforms.

The special case of Theorem 2 in which $\sum_{n=0}^{\infty} a_{n} e^{-\lambda_{n} s}$ is a Dirichlet series, i.e. the case in which the exponents satisfy

$$
0 \leqq \lambda_{0}<\lambda_{1}<\cdots<\lambda_{n} \uparrow \infty
$$

will now be considered.

THEOREM 3. If $f(s)$ is the sum of a Dirichlet series $\sum_{n=0}^{\infty} a_{n} e^{-\lambda_{n} s} a b$ solutely convergent for $\sigma \geqq 0$ and if there exists $a \beta \in \mathfrak{A}$ such that

$$
f(s) g(s) \equiv f(s)\left(\int_{0}^{\infty} e^{-s \mu} \beta(d \mu)\right)=1 \quad(\sigma \geqq 0)
$$

then $\beta$ is purely atomic and its Laplace-Stieltjes transform $g(s)$ is also the sum $\sum_{n=0}^{\infty} b_{n} e^{-\mu_{n} s}$ of a Dirichlet series absolutely convergent for $\sigma \geqq 0$. Such a $g(s)$ will exist if and only if

$$
\inf _{\sigma \geqq 0}|f(s)|>0
$$

and then (if we assume that no $b_{n}=0$ ) the exponents $\mu_{n}$ will all belong to the $J$-module generated by the set $\left\{\lambda_{n}: n \in J\right\}$.

Corollary (Hewitt ANd Williamson). If the function $f(s)$ of Theorem 3 is the sum of an ordinary Dirichlet series $\sum_{n=1}^{\infty} a_{n} n^{-s}$ absolutely convergent for $\sigma \geqq 0$ then $g(s)$, if it exists, is also the sum of an ordinary Dirichlet series $\sum_{n=1}^{\infty} b_{n} n^{-8}$ absolutely convergent for $\sigma \geqq 0$. Again $g(s)$ exists if and only if $f(s)$ satisfies (8).

This corollary is immediate, because the $J$-module generated by the set $L=\{\log n: n=1,2, \cdots\}$ is just $L$.

In order to prove the theorem one has only to show that $g(s)$, when it exists, is the sum of a Dirichlet series. Now the elements of the $J$ module $\Lambda$ generated by $\left\{\lambda_{n}: n \in J\right\}$ can evidently be enumerated as follows: 


$$
0=\nu_{0}<\nu_{1}<\nu_{2}<\cdots<\nu_{n} \uparrow \infty .
$$

Since, by Theorem, 2, each $\mu_{n} \in \Lambda$, it follows that $\sum_{n=0}^{\infty} b_{n} e^{--\mu_{n} 8}$ is a Dirichlet series for a suitable enumeration of the $\mu_{n}$.

3 . In view of the fact that the $J$-modules used in the foregoing discussion are subsemigroups of the additive semigroup $R^{+}$of all nonnegative real numbers, the information provided by Theorems 2 and 3 can be summarized as follows.

For each subsemigroup $\Gamma$ of $R^{+}$, such that $0 \in \Gamma$, let $\mathfrak{A}_{\Gamma}$ denote the set of purely atomic measures in $\mathfrak{A}$ that vanish on sets disjoint from $\Gamma$. Then $\mathfrak{A}_{\Gamma}$ is a Banach subalgebra of $\mathfrak{A}$ (closed with respect to the norm defined by (1)) and an element $\alpha \in \mathfrak{A}_{\Gamma} \subset \mathfrak{A}$ has an inverse in $\mathfrak{A}$ if and only if it has an inverse in $\mathfrak{A}_{\Gamma}$. The spectra $\sigma_{\mathfrak{A}}(\alpha)$ and $\sigma_{\mathfrak{A}_{\Gamma}}(\alpha)$ of any $\alpha \in \mathfrak{A}_{\Gamma}$, qua element of $\mathfrak{A}$ and $\mathfrak{A}_{\Gamma}$ respectively, satisfy

$$
\sigma_{\mathfrak{U}}(\alpha)=\sigma_{\mathfrak{U}_{\Gamma}}(\alpha)=\mathrm{Cl}\{f(s): \sigma \geqq 0\},
$$

where

$$
f(s)=\sum_{n=0}^{\infty} a_{n} e^{-\lambda_{n} s} \quad(\sigma \geqq 0),
$$

and where $\mathrm{Cl}$ denotes closure.

It is clear that the usual theorems (already cited in $\$ 1$ ) on analytic functions of Banach-algebra elements can be applied to the algebras $\mathfrak{A}_{\Gamma}$. Suppose for example that $\alpha \in \mathfrak{R}_{\Gamma}$, that $D \supset \sigma_{\mathfrak{q}_{\Gamma}}(\alpha)$ is an open subset of the Gaussian plane and that $F(z)$ is a complex-valued function defined and holomorphic in $D$. Then because $\mathfrak{A}_{\mathrm{r}}$ is complete there exists a $\beta \in \mathfrak{A}_{\Gamma}$ such that

$$
\sum_{n=0}^{\infty} b_{n} e^{-\mu_{n} s}=F\left(\sum_{n=0}^{\infty} a_{n} e^{-\lambda_{n} s}\right) \quad(\sigma \geqq 0),
$$

where $\sum_{n=0}^{\infty} b_{n} e^{-\mu_{n} s}$ is the Laplace-Stieltjes transform of $\beta$.

The discussion following Theorem 2 shows that even when $f(s)$ possesses zeros in the half-plane $\sigma \geqq 0$ it may be possible to obtain for $[f(s)]^{-1}$ a Laplace-Stieltjes expansion absolutely convergent in the half-plane $\sigma \geqq \tilde{\sigma}$ for some constant $\tilde{\sigma}>0$. When this is the case the following theorem makes it possible to determine the greatest lower bound of the set of admissible values for $\tilde{\sigma}$.

Theorem 4. Let $\alpha \in \mathfrak{A}_{\Gamma}$ and let $f(s) \equiv \sum_{n=0}^{\infty} a_{n} e^{-\lambda_{n s}}\left(\right.$ with $\left.\lambda_{0}=0\right)$ be its Laplace-Stieltjes transform. If $\tilde{\sigma}>0$ and $\tilde{f}(s) \equiv f(s+\tilde{\sigma})($ for $\sigma \geqq 0)$ then $\tilde{f}(s)$ is the Laplace-Stielties transform of an element $\tilde{\alpha} \in \mathfrak{A}_{\Gamma}$ such that 


$$
\sigma_{\mathfrak{M}}(\tilde{\alpha})=\sigma_{\mathfrak{U}_{\Gamma}}(\tilde{\alpha})=\left\{a_{0}\right\} \cup \underset{0<\delta<\tilde{\sigma}}{\bigcap}\{f(s): \sigma>\tilde{\sigma}-\delta\} .
$$

Accordingly, $\tilde{\alpha}$ has an inverse in $\mathfrak{A}$ (and also in $\mathfrak{A}_{\Gamma}$ ) if and only if

(i) $a_{0} \neq 0$, and

(ii) there exists a positive $\delta<\tilde{\sigma}$ such that $\sigma>\tilde{\sigma}-\delta$ implies that $f(s) \neq 0$.

The existence of an $\tilde{\alpha} \in \mathfrak{A}_{\Gamma}$ whose Laplace-Stieltjes transform is $\tilde{f}(s)$ is an immediate consequence of the fact that $\tilde{f}(s)=\sum_{n=0}^{\infty} \tilde{a}_{n} e^{-\lambda_{n} s}$ for $\sigma \geqq 0$, where $\tilde{a}_{n} \equiv a_{n} e^{-\lambda_{n} \tilde{\sigma}}$, so that $\sum_{0}^{\infty}\left|\tilde{a}_{n}\right|<\infty$. Thus, in order to prove the theorem, what we have to show is that

$$
\mathrm{Cl}\{f(s): \sigma \geqq \tilde{\sigma}\}=\left\{a_{0}\right\} \cup \bigcap_{0<\delta<\tilde{\sigma}}\{f(s): \sigma>\tilde{\sigma}-\delta\},
$$

and we begin by showing that for each positive $\delta<\tilde{\sigma}$

$$
\mathrm{Cl}\{f(s): \sigma \geqq \tilde{\sigma}\} \subseteq\left\{a_{0}\right\} \cup\{f(s): \sigma>\tilde{\sigma}-\delta\} .
$$

Suppose that $\left\{s_{n}\right\}$ is any sequence in the half-plane $\sigma \geqq \tilde{\sigma}$ for which $\lim _{n \rightarrow \infty} f\left(s_{n}\right)=\lim _{n \rightarrow \infty} f\left(\sigma_{n}+i t_{n}\right)=w_{0}$ exists. If $\left\{\sigma_{n}\right\}$ is unbounded then it contains a subsequence $\left\{\sigma_{n_{r}}\right\}$ diverging to $\infty$ as $r \rightarrow \infty$. But then we have, as in the proof of Theorem 2,

$$
w_{0}=\lim _{n \rightarrow \infty} f\left(s_{n}\right)=\lim _{r \rightarrow \infty} f\left(s_{n_{r}}\right)=a_{0} .
$$

However, if $\left\{\sigma_{n}\right\}$ is bounded $\left(\tilde{\sigma} \leqq \sigma_{n} \leqq \bar{\sigma}<\infty\right.$ for $\left.n=1,2, \cdots\right)$ we shall see that there exists a point $s^{\prime}$ in the strip $\tilde{\sigma}-\delta<\sigma<\bar{\sigma}+\delta$ for which $f\left(s^{\prime}\right)=w_{0}$, and this will complete the proof of $(10)$.

The series $\sum_{n=0}^{\infty} a_{n} e^{-\lambda_{n} s}$ is uniformly convergent in $\tilde{\sigma}-\delta<\sigma<\bar{\sigma}+\delta$ by Weierstrass's $M$-test (because $\left.\sum_{0}^{\infty}\left|a_{n}\right|<\infty\right) . f(s)$ is therefore in this strip an analytic uniformly almost periodic function in the sense of Bohr. Consequently if $f(s)-w_{0}$ failed to vanish in this strip we should have (by Corollar 1 to Satz 7 of Bohr [2])

$$
\inf _{\tilde{\sigma} \leqq \sigma \leqq \sigma}\left|f(s)-w_{0}\right|>0,
$$

which contradicts the definition of $w_{0}$. Accordingly $f(s)-w_{0}$ does vanish in the strip $\tilde{\sigma}-\delta<\sigma<\bar{\sigma}+\delta$ and so (10) is true.

Since $a_{0} \in \operatorname{Cl}\{f(s): \sigma \geqq \tilde{\sigma}\}$ it will now be enough, in order to prove (9), to show that

$$
E \equiv \bigcap_{0<\delta<\tilde{\sigma}}\{f(s): \sigma>\tilde{\sigma}-\delta\} \subseteq \mathrm{Cl}\{f(s): \sigma \geqq \tilde{\sigma}\} .
$$

If $z_{0} \in E$ then either $z_{0} \in\{f(s): \sigma \geqq \tilde{\sigma}\}$ or else we can find a sequence $\left\{\sigma_{m}+i t_{m}\right\}$ such that $0 \leqq \sigma_{m} \uparrow \tilde{\sigma} \quad(m \rightarrow \infty)$ and $f\left(\sigma_{m}+i t_{m}\right)=z_{0}$ for $m=1,2, \cdots$. Now for each integer $N \geqq 1$ 


$$
\begin{aligned}
\left|z_{0}-f\left(\tilde{\sigma}+i t_{m}\right)\right| & \leqq \sum_{n=1}^{\infty}\left|a_{n}\right|\left|e^{-\lambda_{n} \sigma_{m}}-e^{-\lambda_{n} \tilde{\sigma}}\right| \\
& \leqq \sum_{n=1}^{N}\left|a_{n}\right|\left|e^{-\lambda_{n} \sigma_{m}}-e^{-\lambda_{n} \tilde{\sigma}}\right|+2 \sum_{n=N+1}^{\infty}\left|a_{n}\right| .
\end{aligned}
$$

The final term here is $o(1)$ as $N \rightarrow \infty$. The preceding term is, for fixed $N, o(1)$ as $m \rightarrow \infty$. It follows that $f\left(\tilde{\sigma}+i t_{m}\right) \rightarrow z_{0}$ as $m \rightarrow \infty$ and hence that $z_{0} \in \mathrm{Cl}\{f(s): \sigma \geqq \tilde{\sigma}\}$, as required.

Corollary. If $f(s)$ and $\tilde{\alpha}$ are defined as in Theorem 4 then

$$
\sigma_{\mathfrak{A}}(\tilde{\alpha})=\left\{a_{0}\right\} \cup \mathrm{Cl}\{f(s): \sigma=\tilde{\sigma}\} \cup\{f(s): \sigma>\tilde{\sigma}\} .
$$

An application of Satz 7 of Bohr [2] shows that this corollary remains true if we allow $\tilde{\sigma}=0$.

Theorem 5. Suppose that $f(s) \equiv \sum_{n-0}^{\infty} a_{n} e^{-\lambda_{n} s}$ (with $\lambda_{0}=0$ ) is the Laplace-Stieltjes transform of an element $\alpha \in \mathfrak{A}_{\Gamma}$ such that $a_{0} \neq 0$, and let $\sigma_{0}$ be the greatest lower bound of the numbers $\tilde{\sigma} \geqq 0$ for which the halfplane $\sigma>\tilde{\sigma}$ contains no zeros of the function $f(s)$. Then there exists a series $\sum_{n=0}^{\infty} b_{n} e^{-\mu_{n} s}$, absolutely convergent in the half-plane $\sigma>\sigma_{0}$, satisfying

$$
\left(\sum_{n=0}^{\infty} a_{n} e^{-\lambda_{n} s}\right)\left(\sum_{n=0}^{\infty} b_{n} e^{-\mu_{n} s}\right)=1 \quad \text { for } \sigma>\sigma_{0},
$$

and such that the exponents $\mu_{n}$ all belong to the $J$-module $\Lambda(\subseteq \Gamma)$ generated by $\left\{\lambda_{n}: n \in J\right\}$. If moreover $\sigma_{0}>0$ then $\sum_{n=0}^{\infty}\left|b_{n}\right| e^{-\lambda_{n} \sigma_{0}}=\infty$.

If $\tilde{\sigma}>\sigma_{0}$ then we have only to take $\delta=\left(\tilde{\sigma}-\sigma_{0}\right) / 2$ to see that $f(s)$ satisfies the conditions (i) and (ii) of Theorem 4. Consequently

$$
\frac{1}{\tilde{f}(s)}=\sum_{n=0}^{\infty} \tilde{b}_{n} e^{-\mu_{n} s} \quad(\sigma \geqq 0),
$$

where $\sum_{0}^{\infty}\left|\tilde{b}_{n}\right|<\infty$ and where $\mu_{n} \in \Lambda(n=0,1,2, \cdots)$. If $b_{n} \equiv \tilde{b}_{n} e^{\mu_{n} \tilde{\sigma}}$ then we have

$$
\left(\sum_{n=0}^{\infty} a_{n} e^{-\lambda_{n} s}\right)\left(\sum_{n=0}^{\infty} b_{n} e^{-\mu_{n} s}\right)=1 \quad \text { for } \sigma \geqq \tilde{\sigma} .
$$

This argument works for each $\tilde{\sigma}>\sigma_{0}$, and it is not difficult to show that the series $\sum_{n=0}^{\infty} b_{n} e^{-\mu_{n} s}$ is the same for each such choice of $\tilde{\sigma}$. Consequently the equation (11) holds whenever $\sigma>\sigma_{0}$, and the series $\sum_{n=0}^{\infty} b_{n} e^{-\mu_{n} s}$ is absolutely convergent throughout this half-plane.

Now suppose that $\sigma_{0}>0$ and that, if possible, $\sum_{n=0}^{\infty}\left|b_{n}\right| e^{-\mu_{n} \sigma_{0}}<\infty$. We have just seen that $g(s) \equiv \sum_{n=0}^{\infty} b_{n} e^{-\mu_{n s}}$ satisfies 


$$
f(s) g(s)=1 \quad \text { for } \sigma>\sigma_{0} .
$$

But $f(s)$ and $g(s)$ are continuous throughout $\sigma \geqq \sigma_{0}$ and so this equation holds for $\sigma \geqq \sigma_{0}$. But now, because $g(s)$ is bounded in the halfplane $\sigma \geqq \sigma_{0}$ it must follow that $f(s)$ is bounded away from zero in the same half-plane. But by hypothesis we have, for arbitrarily small $\delta>0$,

$$
0 \in\left\{f(s): \sigma>\sigma_{0}-\delta\right\} .
$$

Putting $\tilde{\sigma}=\sigma_{0}$ in Theorem 4 we arrive immediately at a contradiction, and therefore conclude that $\sum_{n=0}^{\infty}\left|b_{n}\right| e^{-\mu_{n} \sigma_{0}}=\infty$.

The task of determining $\sigma_{0}$ for any given series is of course rarely. an easy one.

Note added November 20, 1957. In conversation, Professor R. Arens has pointed out that my use of Theorem 1 is unnecessary, because a more natural proof of the first part of Theorem 2 is contained in his paper [9]. The reader should however be warned that the fourth sentence on p. 504 to [9], though apparently relevant here, is, as it stands, incorrect (counterexample: the absolutely convergent series $f(s) \equiv 1+\sum_{n=1}^{\infty} 2^{-n} p_{n}^{-s}$, where $p_{1}=2, p_{n}=$ the $n$th prime, has no zeros for $0 \leqq \sigma \leqq \infty$, yet $\left.\inf _{\sigma \geqq 0}|f(s)|=0\right)$.

\section{BIBLIOGRAPHY}

1. R. Arens and A. P. Calderón, Analytic functions of several Banach algebra elements, Ann. of Math. vol. 62 (1955) pp. 204-216.

2. H. Bohr, Zur Theorie der fastperiodischen Funktionen III, Dirichletentwicklung analytischer Funktionen, Acta Math. vol. 47 (1926) pp. 237-281.

3. E. Hewitt and J. H. Williamson, Note on absolutely convergent Dirichlet series, Proc. Amer. Math. Soc. vol. 8 (1957) pp. 863-868.

4. E. Hewitt and H. S. Zuckerman, Finite dimensional convolution algebras, Acta Math. vol. 93 (1955) pp. 67-119.

5. - - The $l_{1}$-algebra of a commutative semigroup, Trans. Amer. Math. Soc. vol. 83 (1956) pp. 70-97.

6. E. Hille and R. S. Phillips, Functional analysis and semi-groups, rev. ed., Amer. Math. Soc. Colloquium Publications, 1957, xii +808 pp.

7. L. H. Loomis, Abstract harmonic analysis, New York, van Nostrand, 1953.

8. R. S. Phillips, Spectral theory for semi-groups of linear operators, Trans. Amer. Math. Soc. vol. 71 (1951) pp. 393-415.

9. R. Arens, $A$ Banach algebra generalization of conformal mappings of the disc, Trans. Amer. Math. Soc. vol. 81 (1956) pp. 501-513.

The Queen's College, Oxford and

YALE UNiversity 